\title{
Adult congenital megacolon with acute fecal obstruction and diabetic nephropathy: A case report
}

\author{
MINGYUAN ZHANG ${ }^{1,2}$ and KEFENG DING ${ }^{1}$ \\ ${ }^{1}$ Colorectal Surgery Department, Second Affiliated Hospital, School of Medicine, Zhejiang University, Hangzhou, \\ Zhejiang 310000; ${ }^{2}$ Department of Gastrointestinal Surgery, Yinzhou Peoples' Hospital, Ningbo, Zhejiang 315000, P.R. China
}

Received November 27, 2018; Accepted June 20, 2019

DOI: $10.3892 /$ etm.2019.7852

\begin{abstract}
Megacolon is a congenital disorder. Adult congenital megacolon (ACM), also known as adult Hirschsprung's disease, is rare and frequently manifests as constipation. ACM is caused by the absence of ganglion cells in the submucosa or myenteric plexus of the bowel. Most patients undergo treatment of megacolon at a young age, but certain patients cannot be treated until they develop bowel obstruction in adulthood. Bowel obstruction in adults always occurs in complex clinical situations and it is frequently combined with comorbidities, including bowel tumors, volvulus, hernias, hypertension or diabetes mellitus. Surgical intervention is always required in such cases. To avoid recurrence, a sufficient amount of bowel should be removed, particularly the aganglionic segment. Furthermore, the patient's general physical condition should be considered pre-operatively by controlling parameters including blood pressure and blood glucose. In the present study, a case of ACM combined with fecal impaction and diabetic nephropathy was presented.
\end{abstract}

\section{Introduction}

Adult congenital megacolon (ACM), also known as adult Hirschsprung's disease (1), is relatively rare; only 300 cases were reported in the literature prior to 2016 (2). ACM frequently presents with bowel obstruction as the initial symptom (3). Affected patients usually have a history of constipation. Bowel obstruction in adults always occurs in complex clinical situations and is frequently combined with comorbidities, including bowel tumors, volvulus, hernias, hypertension or diabetes mellitus. Thus, treatment poses a great clinical challenge (4). Surgical intervention is required, and to avoid recurrence, a

Correspondence to: Professor Kefeng Ding, Colorectal Surgery Department, Second Affiliated Hospital, School of Medicine, Zhejiang University, 88 Jiefang Road, Hangzhou, Zhejiang 310000, P.R. China

E-mail: dingkefeng@126.com

Key words: adult congenital megacolon, bowel obstruction, diabetic nephropathy sufficient amount of bowel should be removed, particularly the aganglionic segment (2). The present study reports on a case of a 56-year-old patient with ACM, fecal impaction and diabetic nephropathy.

\section{Case report}

A 56-year-old male patient with a history of chronic constipation presented to the emergency department of Yinzhou Peoples' Hospital (Ningbo, China) in February 2018. The patient had experienced vague abdominal distention for several days. Prior to admission, chronic bowel obstruction had been diagnosed at another hospital. Auxiliary examination with a barium enema had indicated a dilated bowel loop with a transition zone in the distal sigmoid colon. The patient denied any similar conditions among his family members. The patient's history of constipation started in childhood and remained untreated, but his severe constipation had begun 6 years previously. Furthermore, the patient had a 10-year history of diabetes mellitus and regularly took insulin; his fasting blood glucose concentration was reasonably controlled at 8.0-9.0 $\mathrm{mmol} / 1$.

After admission to our department, the patient's vital signs were normal. Physical examination revealed a soft abdomen with slight distension and tympanic percussion sounds but without local tenderness or rebound pain. The patient's bowel sounds were hypoactive and an anal examination revealed no masses. Laboratory data indicated elevated levels of creatinine, blood urea nitrogen, glucose and potassium. Abdominal computed tomography (CT) revealed a dilated bowel with a typical 'coffee bean sign' (5). The dilated bowel extended from the jejunum to the sigmoid colon and a transition zone was observed in the distal sigmoid colon. The stomach was severely compressed in the left upper quadrant. Large amounts of feces had accumulated inside the colon (Fig. 1).

Initial treatment included gastrointestinal decompression, fluid infusion and insulin to decrease the blood glucose level, which subsequently became well controlled. Laboratory tests indicated that the creatinine and blood urea nitrogen levels decreased several days later and the hyperkalemia resolved. The patient's bowel movements also returned. Abdominal CT suggested that the dilated small bowel had decreased in size at 1 week after admission (Fig. 2). However, the patient's abdomen remained distended without improvement. 
A

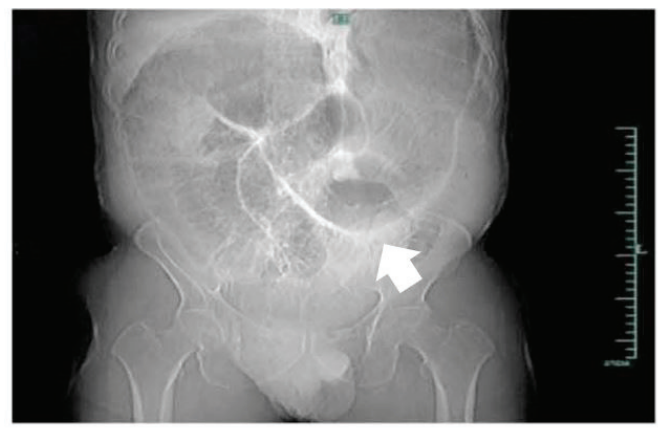

C

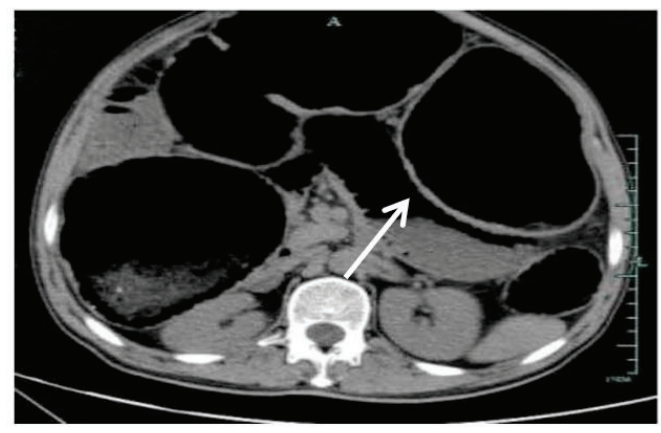

B

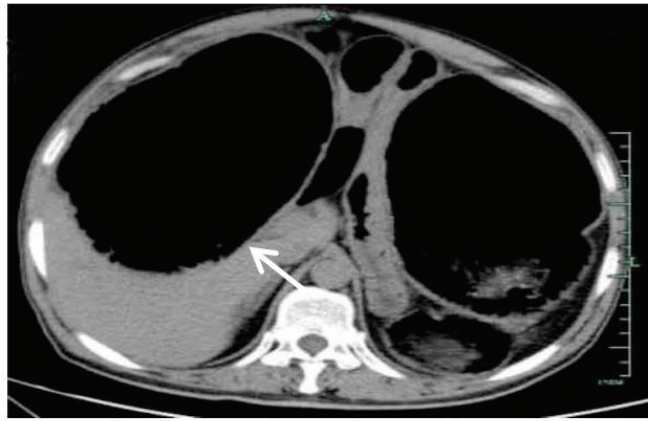

D

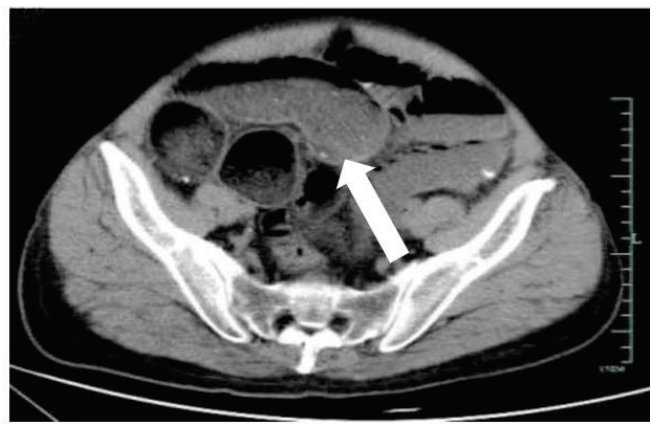

Figure 1. (A) Abdominal CT positioning image revealing a dilated bowel loop and 'coffee bean sign' (arrow). (B) CT transverse view at the epigastrium level revealing a dilated bowel loop of the ascending colon in the upper abdominal cavity (thin arrow). (C) CT transverse view at the epigastrium level revealing the dilated loop of the decending colon (thin arrow). (D) CT transverse view at the pelvic cavity level revealing the dilated loop of the ileum (thick arrow). Every small single counterpart equalled 1 centimeter. Images were captured in the caudal direction. CT, computed tomography.

At 10 days after admission, the patient's abdominal condition suddenly worsened. Severe distention with rebound tenderness was noted and emergency laparotomy was required. During the operation, a severely dilated bowel was encountered, extending from the ascending colon to the sigmoid colon. The most dilated bowel segment was $>15 \mathrm{~cm}$ in diameter (Fig. 3). Numerous hard fecaliths were observed in the descending colon and sigmoid colon, and the colonic lumen was obstructed. No perforation or tumor mass was palpable in the gastrointestinal tract. The portion of colon from the ileocecal junction to the terminal sigmoid colon was removed in a terminal ileostomy. Post-operative pathologic examination revealed the absence of ganglion cells in the submucosa and myenteric plexux of the bowel and confirmed the previous diagnosis (Fig. 4). After the operation, the patient received persistent gastrointestinal decompression, fluid infusion and blood glucose control. Abdominal CT re-examination indicated good recovery (Fig. 5). Post-operative blood tests indicated normal creatinine, blood urea nitrogen and glucose levels. No post-operative complications occurred, the patient was discharged in good physical condition and took follow-ups every 3 months (ongoing), without any disorders.

\section{Discussion}

ACM is a rare condition (6). Rosin et al (7) first reported ACM in 1950. Studies have indicated that affected patients are $>10$ years old at the time of diagnosis (2), the incidence of ACM is $1 / 2,000$ to $1 / 5,000$ in live births, and the sex ratio (male:female) is $3: 1$ to $4: 1(8,9)$. In China, ACM is defined as occurring in patients aged $>14$ years (10) with a colonic condition caused by the absence of ganglion cells in the submucosa or myenteric plexus of the bowel. Chronic constipation or abdominal distension begins to appear at a young age in most patients, but most patients do not undergo treatment, as symptoms are mild. The condition frequently aggravates as the patient ages (11). Bowel obstruction is always the primary symptom at that time. ACM may be misdiagnosed as idiopathic megacolon, Chagas disease, inflammatory bowel disease, bowel volvulus or colorectal tumor.

In China, ACM has been divided into the following six phenotypes according to the affected bowel region: i) Common type: The total rectum and sigmoid colon are affected. ii) Super-short-segment type: Only the terminal rectum is affected. iii) Short-segment type: The terminal rectum to the rectal ampulla is affected. iv) Long-segment type: The rectum to the descending colon and even the transverse colon is affected. v) Total colon type: The rectum, total colon and the last $10 \mathrm{~cm}$ of the terminal ileum are affected. vi) Total bowel type: Further intestinal segments may be affected on the basis of the total colon type. Li and Chen (12) reported on 36 cases of ACM: 23 were of the common type, 7 of the short-segment type, 3 of the super-short-segment type, 1 was of the total colon type and 2 could not be classified. The patient in the present case had total colon-type ACM.

The diagnosis of ACM should be made in considering a history of illness, symptoms, laboratory examination and histopathological examination. This is important, as patients with idiopathic megacolon may also be constipated and have a dilated colon, whereas patients with Chagas disease may also develop an absence of ganglion cells in the submucosal and myenteric layers. Other diseases, including colorectal cancer and bowel volvulus, always manifest as bowel obstruction. According to López Ruiz et al (2), the diagnosis of ACM should be made using barium enema, anorectal manometry and rectal biopsy. Qi and Zhang (9) indicated 

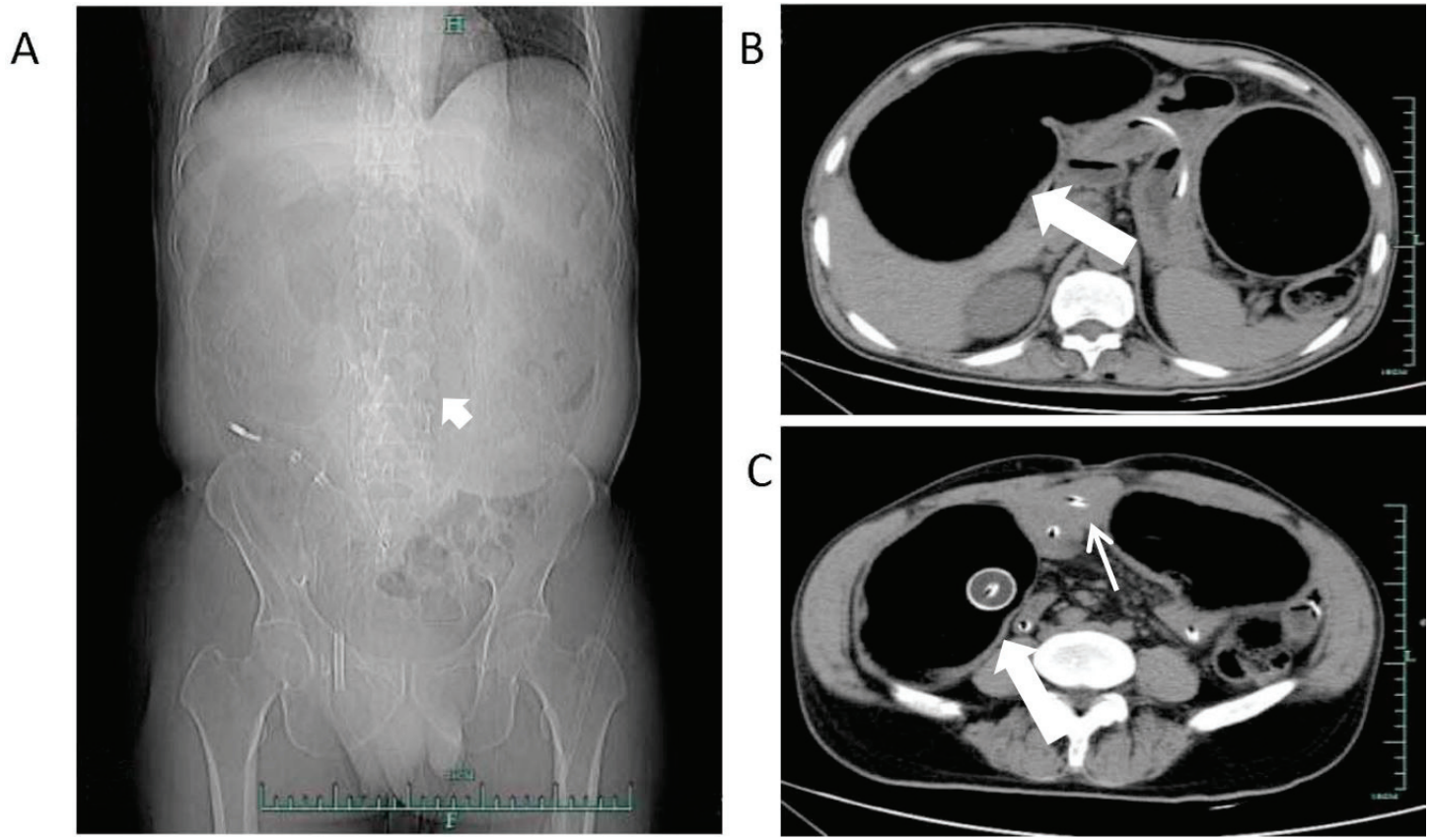

Figure 2. (A) Abdominal CT positioning image (coronal plain) indicating that the dilated intestine loop had substantially decreased in size after treatment and that the previously observed 'coffee bean sign' (arrow) had disappeared. (B) CT transverse view at the epigastrium level revealing that the dilated bowel loop was still present in the ascending colon (thick arrow). (C) CT transverse view at the hypogastrium level revealing the dilated bowel loop in the colon (thick arrow) and the iluem with normal diameter after treatment (thin arrow) between the dilated colon. Every small single counterpart equalled 1 centimeter. Images were captured in the caudal direction. CT, computed tomography.

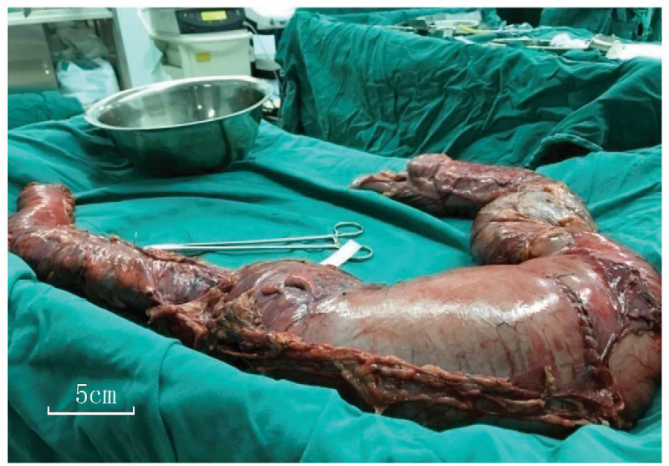

Figure 3. Image of the excised giant colon. The dilated bowel extended from the ascending colon to the sigmoid colon. The most severely dilated section of the bowel was $>15 \mathrm{~cm}$ in diameter.

that a barium enema is one of the most important techniques for diagnosing megacolon, with an accuracy of $\sim 90 \%$. The most important finding on barium enema is the existence of a transition zone between the proximal dilated bowel and distal aganglionic bowel (normal or narrow). A pre-operatively determined radiological transition zone has potential value for identification of the length of resected bowel in patients with ACM, as well as high predictive value for the diagnosis of ACM (13). The level of the radiological transition zone is a useful predictor of actual disease involvement in older patients and infants (13). The absence of a transition zone does not exclude the diagnosis of ACM. The case of the present study did not receive a barium enema, as he had already received one at another hospital prior to admission, but also because of his severely dilated bowel loop, which had a high risk of perforation. Anorectal manometry

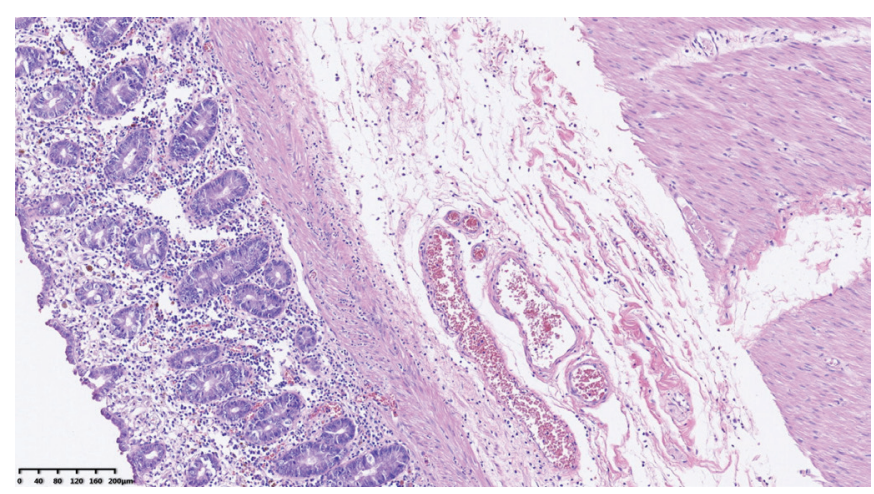

Figure 4. Post-operative pathologic image revealing the absence of ganglion cells in the submucosa and myenteric plexus of the bowel. Hematoxylin and eosin staining at a x100 magnification.

in another hospital prior to admission to Yinzhou Peoples' Hospital indicated a lack of relaxation of the internal anal sphincter in response to rectal distension. In addition, the diagnosis of ACM should be confirmed with a rectal biopsy of a narrow segment (93\% sensitivity and $100 \%$ specificity) (14). In the present case, the patient had a lifelong history of constipation and barium enema at another hospital revealed a dilated bowel loop; rectal biopsy was performed to attain a definitive diagnosis. CT is also useful, as it may reveal the transition zone and may be used to exclude certain other diseases with similar symptoms, including colorectal tumors, volvulus, bowel adhesion, idiopathic megacolon and toxic megacolon. Although abdominal CT may assist in the diagnosis, pathologic examination is the gold standard for obtaining a definitive diagnosis (9). In the present case, the patient finally underwent surgery and the diagnosis of ACM 
A

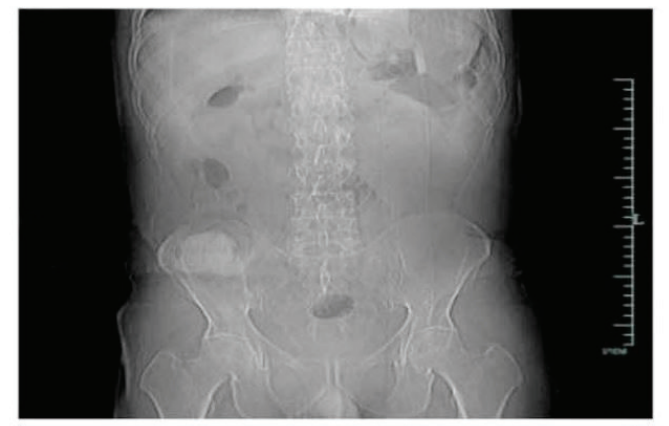

C

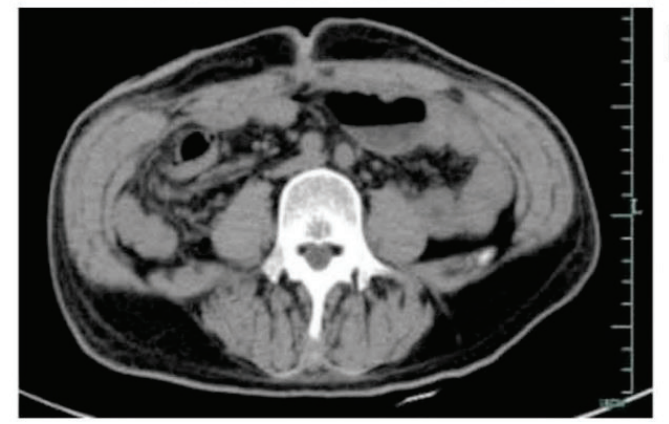

B

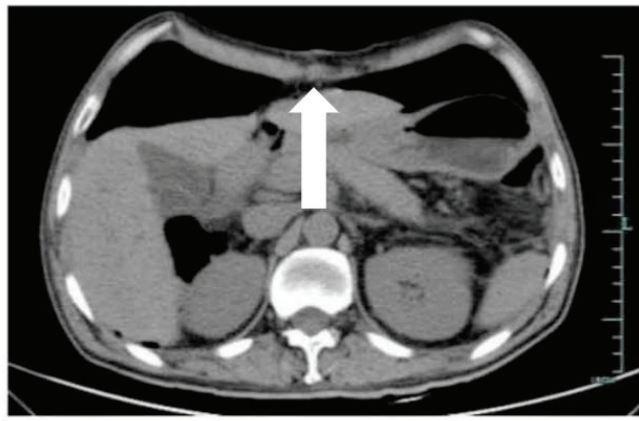

D

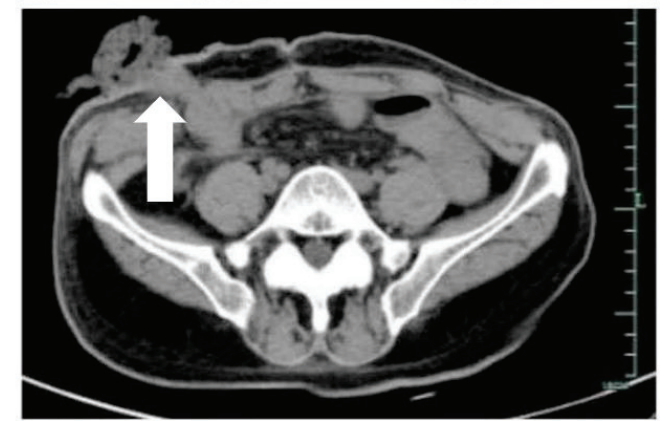

Figure 5. (A) Post-operative abdominal CT positioning image (coronal plain) indicating that the dilated bowel loop had disappeared. (B) CT transverse view at the epigastrium level indicating that the distended abdomen became flattened (thick arrow) and jejunum was of a normal size. (C) $\mathrm{CT}$ transverse view at the hypogastrium level indicating that the previously dilated bowel loop disappeared and ileum was of a normal size. (D) CT transverse view at the pelvic cavity level indicating that the dilated iluem loop disappeared and the iluem diameter became normal (thick arrow). Images were captured in the caudal direction. CT, computed tomography.

was confirmed with the help of pathologic examination, which revealed the absence of ganglion cells in the submucosal and myenteric layers of the colon.

ACM is a special type of neuronal intestinal malformation that not only manifests as ganglion cell deficiency but also as various abnormalities, including hypoganglionosis, immaturity of ganglion cells and non-classifiable dysganglionosis (15). However, the pathogenesis of ACM remains to be fully elucidated. ACM is likely caused by the absence of ganglion cells in the affected segment of bowel, particularly in the distal segment. This is the result of a lack of migration of neuroblasts from the neural crest to the large intestine during the embryonic period. The neural crest is a unique structure that may differentiate into numerous tissue types including ganglion cells. On completion of migration, neural crest cells differentiate into diverse cell types, including neurons and glia of the sensory, sympathetic and parasympathetic ganglia, and neuroendocrine cells. Diseases arising from the neural crest are particularly diverse in their clinical presentations and include digestive, endocrinologic, neurological and cutaneous symptoms. To contribute to enteric nervous system development, neural crest cells must leave the neural tube, migrate to and enter the gut, and then begin their migration along the hindgut. These events are regulated by molecular and cellular mechanisms that are not completely understood. Other proposed mechanisms include defects in neuroblast differentiation and accelerated destruction of ganglion cells in the intestine (16). Mechanisms including RET gene mutation $(17,18)$ have been widely accepted. The RET proto-oncogene is the major gene causing this type of disease; however, RET mutations only account for 20 and $50 \%$ of sporadic and familial cases, respectively (19). Different human and animal genetic studies have identified six associated genes: The RET proto-oncogene, the genes encoding SOX10, endothelin-converting enzyme, endothelin B receptor gene, endothelin 3 and glial cell line-derived neurotrophic factor. Microenvironmental factors also have a role in the pathogenesis of aganglionosis (19). In the present case, it was suggested to the patient to undergo RET gene detection analysis; however, he declined due to limited economic resources.

Surgical intervention is always a necessary part of treatment. López Ruiz et al (2) reported that the surgical approach depends on the length of the aganglionic area, the length and reversibility of the colonic dilatation and the nutritional status of the patient. Usually, a longer duration of the obstruction is associated with more complications. This is due to a large amount of stool accumulating in the bowel cavity, leading to bowel dilation and thereby increasing the risk of bowel necrosis, perforation, abdominal infection and volvulus. Therefore, surgical decompression must be performed. Sufficient decompression may decrease post-operative complications. A shorter duration of bowel obstruction is associated with a lower probability of bacterial translocation. In the present case, the patient underwent gastrointestinal decompression upon admission, and surgery was then performed. The patient also had a 10-year history of diabetes mellitus. Upon admission to our department, the patient's blood glucose, creatinine, blood urea nitrogen and potassium levels were elevated; therefore, an endocrinology consultation was immediately performed to guide treatment. No post-operative complications occurred. Selection of the surgical method should depend on the patient's actual condition and the surgeon's skills. The length of the resected bowel is not only determined using radiological tests but also by pathologic examination. In the present case, the patient had a long history of illness, and the dilated bowel 
extended from the ileocecal junction to the sigmoid colon; this is why the patient underwent total colectomy with end ileostomy, as pathologic examination indicated a normal incisal margin. With advancements in surgical skills and equipment, novel treatment methods, including transanal surgery and laparoscopic megacolonectomy, are being increasingly accepted by surgeons (20). Transanal surgery has the advantage of minimal invasion (21), whereas laparoscopic megacolonectomy changes a traditional operation into laparoscopy, thereby greatly decreasing operative injury, and it is suited for any type of megacolon. This method has therefore become popular for the surgical treatment of ACM (22).

In summary, $\mathrm{ACM}$ is a rare but fatal alimentary tract malformation with a complex etiopathogenesis and presentation, and associated complications are usually present. Surgeons should make an accurate diagnosis based on the history of illness, symptoms, laboratory test results and radiologic findings. Surgical intervention is always needed, and prior to surgery, the patient's general condition (blood pressure, blood glucose and other parameters) should be regulated to decrease the risk of post-operative complications. A sufficient amount of bowel should be removed, including the aganglionic segment. The length of the resected bowel should be determined using not only radiologic tests but also frozen-section pathologic examination. Emergency total colectomy with end ileostomy is the gold standard procedure (23). Patients with ACM require particular attention, and timely diagnosis and individualized treatment may accelerate the recovery process.

\section{Acknowledgements}

The authors thank Dr Angela Morben for editing the English draft of this manuscript.

\section{Funding}

No funding was received.

\section{Availability of data and materials}

The datasets used and/or analyzed during the present study are available from the corresponding author on reasonable request.

\section{Authors' contributions}

KD conceived the case report. MZ treated the patient, made substantial contributions to the acquisition of data, and was involved in drafting the manuscript and revising it critically.

\section{Ethics approval and consent to participate}

Not applicable.

\section{Patient consent for publication}

The patient provided written informed consent for publication.

\section{Competing interests}

The authors declare that they have no competing interets.

\section{References}

1. Fortea-Sanchís C, Martínez-Ramos D, Rivadulla-Serrano I, Daroca-José JM, Paiva-Coronel G and Salvador-Sanchís JL: Hirschprung's disease in adults. Rev Esp Enferm Dig 103: 150-151, 2011.

2. López Ruiz JA, Tallón Aguilar L, Sánchez Moreno L, López Pérez J, Pareja Ciuró F, Oliva Mompeán F and Padillo Ruiz FJ: Hirschsprung disease with debut in adult age as acute intestinal obstruction: Case report. Rev Esp Enferm Dig 108: 742-746, 2016.

3. Lou Z, Meng RG, Yu ED, Liu LJ, Hao LQ, Wang HT and Fu CG: Diagnosis and surgical treatment of adult Hirschsprung's disease. Zhonghua Wei Chang Wai Ke Za Zhi 8: 304-305, 2005 (In Chinese)

4. Doodnath R and Puri P: A systematic review and meta-analysis of Hirschsprung's disease presenting after childhood. Pediatr Surg Int 26: 1107-1110, 2010.

5. Wu S, Sun X, Yu Y and Shen Y: Hirschsprung's disease-related sigmoid vulvulus complicated with refractory hypertension. Am J Case Rep 19: 467-471, 2018.

6. Shitta AH, Ugwu BT, Peter SD, Ozoilo KN, Adighije PF and Omolabake BI: Hirschsprung's disease in an adult: A case report. J West Afr Coll Surg 4: 121-126, 2014.

7. Rosin JD, Bargen JA and Waugh JM: Congenital megacolon of a man 54 years of age: Report of case. Proc Staff Meet Mayo Clin 25: 710-715, 1950.

8. Qiu JF, Shi YJ, Hu L, Fang L, Wang HF and Zhang MC: Adult Hirschsprung's disease: Report of four cases. Int J Clin Exp Pathol 6: 1624-1630, 2013.

9. Qi Z and Zhang W: Adult congenital megacolon with bowel obstruction: A case report. Chin J Gastroenterol Hepatolt 26: 1131-1132, 2017 (In Chinese).

10. Ding SQ, Chen YT, Ding YJ, Fang L, Wang HF and Zhang MC: Diagnosis and surgical management of adult Hirschsprung disease. Zhonghua Wei Chang Wai Ke Za Zhi 9: 53-55, 2006 (In Chinese).

11. Kim HJ, Kim AY, Lee CW, Yu CS, Kim JS, Kim PN, Lee MG and Ha HK: Hirschsprung disease and hypoganglionosis in adults: Radiologic findings and differentiation. Radiology 247: 428-434, 2008.

12. Li F and Chen Y: Diagnosis and surgical treatment of adult Hirschsprung's disease. Chin J Gen Surg 9: 452-454, 2000 (In Chinese).

13. Chen X, Xiaojuan W, Zhang H, Jiao C, Yu K, Zhu T and Feng J: Diagnostic value of the preoperatively detected radiological transition zone in Hirschsprung's disease. Pediatr Surg Int 33: 581-586, 2017.

14. Arshad A, Powell C and Tighe MP: Hirschsprung's disease. BMJ 345: e5521, 2012.

15. Holschneiderd AM, Meier-Ruge W and Ure BM: Hirschsprung's disease and allied disorders-a review. Eur J Pediatr Surg 4: 260-266, 1994.

16. Fu CG, Muto $\mathrm{T}$, Masaki $\mathrm{T}$ and Nagawa $\mathrm{H}$ : Zonal adult Hirschsprung's disease. Gut 39: 765-767, 1996.

17. Edery P, Lyonnet S, Muligan LM, Pelet A, Dow E, Abel L, Holder S, Nihoul-Fékété C, Ponder BA and Munnich A: Mutations of the RET proto-oncogene in Hirschsprung disease. Nature 367: 378-380, 1994.

18. Romeo G, Ronchetto P, Yin L, Barone V, Seri M, Ceccherini I, Pasini B, Bocciardi R, Lerone M, Kääriäinen H, et al: Point mutations affecting the tyrosine kinase domain of the RET proto-oncogene in Hirschsprung's disease. Nature 367: 377-378, 1994.

19. Martuccielb G, Ceccherim I, Lerong M and Jasonni V: Pathogenesis of Hirschsprung's disease. J Pediatr Surg 55: 1017-1025, 2000.

20. He X, Xu D and Li L: Congenital megacolon with Laporoscopicassisted treatment: 20 cases report. J Laporoscopic surg 14: 55-56, 2009.

21. Wang G: The treatment progession and micro invasive surgery of Congenital megacolon. Chin J Pediatr surg 23: 103-104, 2003 (In Chinese).

22. Jarry J and Faucheron JL: Laparoscopic rectosigmoid resection with transanal colonic pull-through and delayed coloanal anastomosis: A new approach to adult Hirschsprung disease. Dis Colon Rectum 54: 1313-1319, 2011.

23. Miniello S, Marzaioli R, Balzanelli MG, Dantona C, Lippolis AS, Barnabà $\mathrm{D}$ and Nacchiero $\mathrm{M}$ : Toxic megacolon in ulcerative rectocolitis. Current trends in clinical evaluation, diagnosis and treatment. Ann Ital Chir 85: 45-49, 2014. 